Bull. Austral. Math. Soc.

$35 \mathrm{~A} 15,35 \mathrm{~B} 35,35 \mathrm{~J} 20,47 \mathrm{~N} 15$

VOL. 75 (2007) [381-395]

\title{
ON THE NONLINEAR DIRICHLET PROBLEM WITH $p(x)$-LAPLACIAN
}

\section{Marek Galewski and Marek Plócienniczak}

Using a dual variational method which we develop, we show the existence and stability of solutions for a family of Dirichlet problems

$$
\begin{gathered}
-\operatorname{div}(\nu(x, \nabla u(x)))=F_{u}^{k}(x, u(x)), \\
\left.u(x)\right|_{\partial \Omega}=0, u \in W_{0}^{1, p(x)}(\Omega),
\end{gathered}
$$

$k=0,1, \ldots$ in a bounded domain in $\mathbb{R}^{N}$ and with the nonlinearity satisfying some general growth conditions. The assumptions put on $v$ are satisfied by $p(x)$-Laplacian operators.

\section{INTRODUCTION}

In the paper we consider a problem

$$
\begin{gathered}
-\operatorname{div}(\nu(x, \nabla u(x)))=F_{u}^{k}(x, u(x)), \\
\left.u(x)\right|_{\partial \Omega}=0, u \in W_{0}^{1, p(x)}(\Omega)
\end{gathered}
$$

for $k=0,1, \ldots$ and with suitable assumptions on $\mathcal{V}$ which are valid for the $p(x)$ Laplacian operator. Following some ideas from [11] we construct a dual variational method which applies to more general type of nonlinearities than those that are subject to a Palais-Smale type condition. We relate critical values and critical points to the action functional for which (1.1) is the Euler-Lagrange equation and the dual action functional (introduced in the paper and different from the Clarke dual functional) on specially constructed subsets of their domains.

A variational approach concerning existence results for a class of problems involving critical and subcritical growth is shown in [2]. Critical point theory in certain Sobolev spaces is used to obtain existence and multiplicity results in sublinear and superlinear cases as well [5], where the problem is written as

$$
-\operatorname{div}\left(|\nabla u|^{p(x)-2} \nabla u\right)+|u|^{p(x)-2} u=f(x, u)
$$

Received 24th October, 2006

Copyright Clearance Centre, Inc. Serial-fee code: 0004-9727/07 \$A2.00+0.00. 
$u \in W^{1, p(x)}\left(\mathbb{R}^{N}\right)$. Standard variational arguments in Sobolev spaces with variable exponents are used to show the existence of radial solutions $u \in W^{1, p(x)}\left(\mathbb{R}^{N}\right)$ with $\sup _{x \in \mathbb{R}^{N}} p(x)<N$ in [17] where we assert $\inf _{x \in \Omega} p(x)>N$ in a bounded domain. The singularity of positive radial solutions to

$$
-\operatorname{div}\left(|\nabla u|^{p(x)-2} \nabla u\right)=f(x, u)
$$

with $p, f$ radial in $x, f$ continuous and growth speed rate less than $N(p(x)-1) /(N$ $-p(x))$ as $u \rightarrow \infty$ one can find in [16].

Such problems as ours are studied in $[3,7]$ and these can be applied in elastic mechanics and electrorheological fluid dynamics. Although in both [3] and [7] the problems are not studied in the form which we investigate. See $[13,18]$ and references therein. In [7] the authors consider the problem

$$
\begin{gathered}
-\operatorname{div}\left(|\nabla u(x)|^{p(x)-2} \nabla u(x)\right)=F_{x}(x, u(x)), \\
\left.u(x)\right|_{\partial \Omega}=0, u \in W_{0}^{1, p(x)}(\Omega)
\end{gathered}
$$

where $\Omega \subset \mathbb{R}^{N}$ is a bounded region and $W_{0}^{1, p(x)}(\Omega)$ denotes the generalised Orlicz-Sobolev space, (see $[6,8]$ ), while in [3] the equation studied is

$$
\begin{gathered}
-\operatorname{div}\left(a(x)|\nabla u(x)|^{p(x)-2} \nabla u(x)+b(x)|u(x)|^{p(x)-2}\right)=F_{x}(x, u(x)), \\
\left.u(x)\right|_{\partial \Omega}=0, u \in W_{0}^{1, p(x)}(\Omega)
\end{gathered}
$$

with $a, b \in L^{\infty}(\Omega)$ such that $a(x) \geqslant a_{0}>0$ and $b(x) \geqslant b_{0} \geqslant 0$ almost everywhere on $\Omega$.

Both papers deal with sublinear and superlinear nonlinearities. In the sublinear case the direct method of the calculus of variations is used while in the superlinear case they apply the mountain pass geometry by showing that a type of Palais-Smale condition is satisfied.

Our method is based on investigating the primal action functional; that is, a functional for which the original problem is the Euler-lagrange equation and the suitably constructed dual one. The duality relations between the two functionals, derived with the aid of Fenchel-Young conjugacy [4] allows for relating the critical values to both functionals on suitably constructed subsets of their domains. Later we relate the relevant critical points at which the critical values are obtained. When this is done we are in a position to chose a minimising sequence and prove that it is convergent to a solution to the original problem.

The novelty of our approach allows also for considering the stability of solutions. Following some general framework for studying stability of solutions for variational problems in sublinear case [12, 14], [15] we provide suitable results for the Dirichlet problem involving $p(x)$-Laplacian problem also. The approach we use has been sketched in the first author's previous work [10]. 


\section{THE ASSUMPTIONS AND AUXILIARY RESUlTS}

In what follows by $C_{S}$ we denote the best Sobolev constant

$$
\|u\|_{p(x)} \leqslant C_{S}\|\nabla u\|_{p(x)} \text { for all } u \in W_{0}^{1, p(x)}(\Omega) .
$$

Since $W_{0}^{1, p(x)}(\Omega)$ is continuously embedded into $W_{0}^{1, p^{-}}(\Omega),[6]$ and by the Sobolev Imbedding Theorem [1], we denote by $C_{1}$ and $C_{2}$ the following constants

$$
\begin{gathered}
\|\nabla u\|_{p^{-}} \leqslant C_{1}\|\nabla u\|_{p(x)}, \\
\max _{x \in \Omega}|u(x)| \leqslant C_{2}\|\nabla u\|_{p^{-}} \text {for all } u \in W_{0}^{1, p^{-}}(\Omega) .
\end{gathered}
$$

Here $p, q \in C(\bar{\Omega}), 1 / p(x)+1 / q(x)=1$ for $x \in \Omega$ and $p^{-}=\inf _{x \in \Omega} p(x), p^{+}=\sup _{x \in \Omega} p(x)$, $p^{-}>N, N>2$.

We also assume

F1 $\operatorname{vol}(\Omega) \leqslant\left(1 / p^{-}+1 / q^{-}\right)^{-1} ;$ there exist positive numbers $d_{0}, d_{1}, d_{2}, \ldots$ such that $C_{1} C_{2} \leqslant d_{k} \leqslant d_{0}$ for $k=1,2, \ldots$. For all $k=0,1,2, \ldots: F_{u}^{k}\left(\cdot, d_{k}\right)$, $F_{u}^{k}\left(\cdot,-d_{k}\right) \in L^{\infty}(\Omega)$ and

$$
\begin{gathered}
C_{1} C_{2} C_{S} \underset{x \in \Omega}{\operatorname{esssup}}\left|F_{u}^{k}\left(x, d_{k}\right)\right| \leqslant a_{0} d_{k}, \\
C_{1} C_{2} C_{S} \underset{x \in \Omega}{\operatorname{ess} \sup }\left|F_{u}^{k}\left(x,-d_{k}\right)\right| \leqslant a_{0} d_{k} .
\end{gathered}
$$

F2 There exists positive number $d>d_{0}$ such that for all $k=0,1,2, \ldots$ and $I=[-d, d]: F_{u}^{k}(\cdot, d), F_{u}^{k}(\cdot,-d) \in L^{\infty}(\Omega), F^{k}: \Omega \times I \rightarrow \mathbb{R}$ are Caratheodory functions and convex in $u$ for almost everywhere $x \in \Omega, F_{u}^{k}: \Omega \times I \rightarrow \mathbb{R}$ are Caratheodory functions, $F^{k}(x, u):=+\infty$ for $(x, u) \in \Omega \times(\mathbb{R}-I)$.

F3 $F_{u}^{k}(x, 0) \neq 0$, for almost everywhere $x \in \Omega, x \mapsto F^{k}(x, 0)$ and $x \mapsto\left(F^{k}\right)^{*}(x, 0)$ are integrable on $\Omega, k=0,1, \ldots$.

F4 $V: \Omega \times \mathbb{R}^{N} \rightarrow \mathbb{R}$ is a Caratheodory function, convex in the second variable, $\mathcal{V}=\left(V_{u_{1}}, V_{u_{2}}, \ldots, V_{u_{N}}\right): \Omega \times \mathbb{R}^{N} \rightarrow \mathbf{R}, V_{u_{i}}, i=1, \ldots, N$ are Caratheodory functions. $\quad \mathcal{V}(x, 0)=0, V(x, 0)=0$ almost everywhere on $\Omega$ and $x \mapsto V(x, 0)$ is integrable on $\Omega$ for almost everywhere $x \in \Omega$.

F5 There exists a constant $a_{0}$ such that

$$
a_{0} \int_{\Omega}|\nabla u(x)|^{p(x)} d x \leqslant \int_{\Omega}|\mathcal{V}(x, \nabla u(x)) \nabla u(x)| d x
$$

for all $u \in W_{0}^{1, p(x)}(\Omega)$.

Here $\left(F^{k}\right)^{*}$ denotes the Fenchel-Young conjugate of $F^{k}$, see [4]. Now for each $k=0,1, \ldots F^{k}: \Omega \times \mathbb{R} \rightarrow \mathbb{R}$ is convex and lower semi-continuous. 
Relation (1.1) is the Euler-Lagrange equation for a functional $J_{k}: W_{0}^{1, p(x)}(\Omega) \rightarrow \mathbb{R}$ for $k=0,1, \ldots$

$$
J_{k}(u)=\int_{\Omega} V(x, \nabla u(x)) d x-\int_{\Omega} F^{k}(x, u(x)) d x .
$$

Let $W=\left\{v \in L^{q(x)}(\Omega): \operatorname{div} v \in L^{q(x)}(\Omega)\right\}$. The dual functional $J_{D_{k}}: W \rightarrow \mathbb{R}$ reads

$$
J_{D_{k}}(v)=\int_{\Omega}\left(F^{k}\right)^{*}(x,-\operatorname{div} v(x)) d x-\int_{\Omega} V^{*}(x, v(x)) d x
$$

for $k=0,1, \ldots$ We assume that the operator $L: W_{0}^{1, p(x)}(\Omega) \rightarrow\left(W_{0}^{1, p(x)}(\Omega)\right)^{*}$ given by

$$
\langle L(g), h\rangle=\int_{\Omega} \mathcal{V}(x, \nabla g(x)) \nabla h(x) d x
$$

for all $g, h \in W_{0}^{1, p(x)}(\Omega)$ has the following properties

F6 $L$ is a continuous, bounded and strictly monotone operator having $\left(S_{+}\right)$ property and being a homeomorphism.

Property $\left(S_{+}\right)$means that $u_{n} \rightarrow u$ in $W_{0}^{1, p(x)}(\Omega)$ provided $u_{n} \rightarrow u$ in $W_{0}^{1, p(x)}(\Omega)$ and $\limsup _{n \rightarrow \infty}\left\langle L\left(u_{n}\right)-L(u), u_{n}-u\right\rangle \leqslant 0$. Therefore

LEMMA 2.1. For any fixed $f \in L^{\infty}(\Omega)$ the Dirichlet problem

$$
\begin{aligned}
& -\operatorname{div}(\nu(x, \nabla u(x)))=f(x) \\
& \left.u(x)\right|_{\partial \Omega}=0, u \in W_{0}^{1, p(x)}(\Omega)
\end{aligned}
$$

has a unique weak solution.

Now we shall construct certain nonlinear subsets of spaces $W_{0}^{1, p(x)}(\Omega)$ and $W$ on which we shall look for critical points and critical values of the action and dual action functional. Having established the relationship between the relevant critical points we get the solution to (1.1). For $k=0,1, \ldots$ we define

$$
\widetilde{X_{k}}=\left\{u \in W_{0}^{1, p(x)}(\Omega):\|\nabla u\|_{p(x)} \leqslant \frac{d_{k}}{C_{1} C_{2}},|u(x)| \leqslant d_{k} \text { for all } x \in \Omega\right\}
$$

We consider a set $X_{k}$ such that for all $u \in X_{k}$ the relation

$$
\begin{gathered}
-\operatorname{div}(\mathcal{V}(x, \nabla \tilde{u}(x)))=F_{u}^{k}(x, u(x)) \\
\left.\widetilde{u}(x)\right|_{\partial \Omega}=0, u \in W_{0}^{1, p(x)}(\Omega)
\end{gathered}
$$

implies $\tilde{u} \in X_{k}$. 
Proposition 2.2. $X_{k}=\widetilde{X}_{k}$.

Proof: Fix $k=0,1, \ldots$ We take any $h \in \widetilde{X_{k}}$. The solution $u \in W_{0}^{1, p(x)}(\Omega)$ to

$$
\begin{gathered}
-\operatorname{div}(\mathcal{V}(x, \nabla u(x)))=F_{u}^{k}(x, h(x)) \\
\left.u(x)\right|_{\partial \Omega}=0
\end{gathered}
$$

exists by Lemma 2.1. Multiplying (2.5) by $u(x)$, calculating integrals and using assumptions (F5) and (F1) we have

$$
\begin{aligned}
a_{0} \int_{\Omega}|\nabla u(x)|^{p(x)} d x & \leqslant \int_{\Omega}|\mathcal{V}(x, \nabla u(x)) \nabla u(x)| d x \\
& \leqslant \max \left\{\int_{\Omega}\left|F_{u}^{k}\left(x, d_{k}\right) u(x)\right| d x, \int_{\Omega}\left|F_{u}^{k}\left(x,-d_{k}\right) u(x)\right| d x\right\} \\
& \leqslant\left(\frac{1}{p^{-}}+\frac{1}{q^{-}}\right) \operatorname{vol}(\Omega) \cdot \frac{a_{0} d_{k}}{C_{1} C_{2} C_{S}}\|u\|_{p(x) .}
\end{aligned}
$$

So

$$
\int_{\Omega}|\nabla u(x)|^{p(x)} d x \leqslant \frac{d_{k}}{C_{1} C_{2}}\|\nabla u\|_{p(x)}
$$

If $\int_{\Omega}|\nabla u(x)|^{p(x)} d x \leqslant 1$ then $\|\nabla u\|_{p(x)} \leqslant 1 \leqslant\left(d_{k}\right) /\left(C_{1} C_{2}\right)$.

If $\int_{\Omega}|\nabla u(x)|^{p(x)} d x \geqslant 1$ we get $\int_{\Omega}|\nabla u(x)|^{p(x)} d x \geqslant\|\nabla u\|_{p(x)}^{p^{-}}$so we have

$$
\|\nabla u\|_{p(x)} \leqslant\|\nabla u\|_{p(x)}^{p^{-}-1} \leqslant \frac{d_{k}}{C_{1} C_{2}} .
$$

Using inequalities (2.2) and (2.1) we obtain

$$
\frac{d_{k}}{C_{1} C_{2}} \geqslant\|\nabla u\|_{p(x)} \geqslant \frac{1}{C_{1}}\|\nabla u\|_{p^{-}} \geqslant \frac{1}{C_{1} C_{2}} \max _{x \in \Omega}|u(x)| \geqslant \frac{1}{C_{1} C_{2}}|u(x)|
$$

for all $x \in \Omega$. Therefore $|u(x)| \leqslant d_{k}$. Thus $u \in \widetilde{X_{k}}$ and we may put $X_{k}=\widetilde{X_{k}}$.

The dual functional $J_{D_{k}}$ will be considered on a set

$$
\begin{aligned}
& X_{k}^{d}=\left\{v \in W: \exists u, \widetilde{u} \in X_{k}\right. \text { related by (2.4) such that } \\
& \left.\qquad-\operatorname{div} v(x)=F_{u}^{k}(x, u(x)) \text { and } v(x)=\mathcal{V}(x, \widetilde{u}(x))\right\} .
\end{aligned}
$$

$J_{k}$ and $J_{D_{k}}$ are well defined on $X_{k}$ and $X_{k}^{d}$ due to the following

LEMMA 2.3. For any $k=0,1, \ldots$ there exist constants $\gamma_{k}, \eta_{k}>0$ such that $\int_{\Omega}\left|F^{k}(x, u(x))\right| d x \leqslant \gamma_{k}$ for all $u \in X_{k}$ and $\left|\int_{\Omega}\left(F^{k}\right)^{*}(x,-\operatorname{div} v(x)) d x\right| \leqslant \eta_{k}$ for all $v \in X_{k}^{d}$. 
Proof: We have from (F1) and (F3) for almost everywhere $x \in \Omega$, since $u \in X_{k}$, that

$$
\begin{aligned}
\int_{\Omega}\left|F^{k}(x, u(x))\right| d x & \leqslant \int_{\Omega}\left|F^{k}(x, 0)\right| d x+\int_{\Omega}\left|F_{u}^{k}(x, u(x))\right||u(x)| d x \\
& \leqslant \int_{\Omega}\left|F^{k}(x, 0)\right| d x+d_{k} \int_{\Omega}\left|F_{u}^{k}\left(x, d_{k}\right)\right| d x=\gamma_{k} .
\end{aligned}
$$

Now only the boundedness of the integral $\int_{\Omega}\left|\left(F^{k}\right)^{*}(x,-\operatorname{div} v(x))\right| d x$ remains to be shown. Since $\left(F^{k}\right)^{*}$ is convex, we have for almost everywhere $x \in \Omega$ that

$$
-F^{k}(x, 0) \leqslant\left(F^{k}\right)^{*}(x,-\operatorname{div} v(x)) \leqslant\left(F^{k}\right)^{*}(x, 0)+\xi(x)(-\operatorname{div} v(x)),
$$

where $\left.\xi(x) \in \alpha F^{k}\right)^{*}(x,-\operatorname{div} v(x))$. From the definition of $X_{k}^{d}$ it follows that there exists $u \in X_{k}$ such that

$$
-\operatorname{div} v(x)=F_{u}^{k}(x, u(x))
$$

By (F2) and (F3)

$$
\begin{aligned}
-\int_{\Omega} F^{k}(x, 0) & d x \leqslant \int_{\Omega}\left|\left(F^{k}\right)^{*}(x,-\operatorname{div} v(x))\right| d x \leqslant \int_{\Omega}\left|\left(F^{k}\right)^{*}(x, 0)\right| d x \\
+ & \int_{\Omega}|u(x)| \cdot|-\operatorname{div} v(x)| d x \leqslant \int_{\Omega}\left|\left(F^{k}\right)^{*}(x, 0)\right| d x+d_{k} \int_{\Omega}\left|F_{u}^{k}\left(x, d_{k}\right)\right| d x .
\end{aligned}
$$

Hence $\eta_{k}=\max \left\{-\int_{\Omega} F^{k}(x, 0) d x, \int_{\Omega}\left|\left(F^{k}\right)^{*}(x, 0)\right| d x+d_{k} \int_{\Omega}\left|F_{u}^{k}\left(x, d_{k}\right)\right| d x\right\}$.

Theorem 2.4. (Duality Principle)

$$
\inf _{u \in X_{k}} J_{k}(u)=\inf _{v \in X_{k}^{d}} J_{D_{k}}(v)
$$

Proof: We fix $k=0,1, \ldots$ and define a functional $J_{k}^{\#}: X_{k} \times X_{k}^{d} \rightarrow \mathbb{R}$,

$$
\begin{aligned}
J_{k}^{\#}(u, v)=-\int_{\Omega} u(x)(-\operatorname{div} v(x)) d x & +\int_{\Omega} V(x, \nabla u(x)) d x \\
& +\int_{\Omega}\left(F^{k}\right)^{*}(x,-\operatorname{div} v(x)) d x
\end{aligned}
$$

We observe that for any $u \in X_{k}$

$$
\inf _{v \in X_{k}^{d}} J_{k}^{\#}(u, v)=J_{k}(u)
$$


Fix $u \in X_{k}$.

$$
\begin{aligned}
\inf _{v \in X_{k}^{d}} J_{k}^{\#}(u, v)=-\sup _{v \in X_{k}^{d}}\left\{\int_{\Omega} u(x)(-\operatorname{div} v(x)) d x\right. & \\
& \left.-\int_{\Omega}\left(F^{k}\right)^{*}(x,-\operatorname{div} v(x)) d x\right\}+\int_{\Omega} V(x, \nabla u(x)) d x \\
\geqslant- & \sup _{f \in L^{q(x)}(\Omega)}\left\{\int_{\Omega} u(x) f(x) d x-\int_{\Omega}\left(F^{k}\right)^{*}(x, f(x)) d x\right\} \\
& \quad+\int_{\Omega} V(x, \nabla u(x)) d x \\
=- & -\int_{\Omega} F^{k}(x, u(x)) d x+\int_{\Omega} V(x, \nabla u(x)) d x=J_{k}(u) .
\end{aligned}
$$

On the other hand, for any $u \in X_{k}$ there exists $\tilde{v} \in X_{k}^{d}$ such that

so

$$
\begin{gathered}
-\operatorname{div} \widetilde{v}(x)=F_{u}^{k}(x, u(x)) \\
\int_{\Omega} F^{k}(x, u(x)) d x+\int_{\Omega}\left(F^{k}\right)^{*}(x,-\operatorname{div} \widetilde{v}(x)) d x=\int_{\Omega} u(x) \operatorname{div} \tilde{v}(x) d x,
\end{gathered}
$$

$$
\begin{aligned}
J_{k}(u) & =\int_{\Omega} V(x, \nabla u(x)) d x+\int_{\Omega}\left(F^{k}\right)^{*}(x,-\operatorname{div} \widetilde{v}(x)) d x-\int_{\Omega} u(x) \operatorname{div} \widetilde{v}(x) d x \\
& =J_{k}^{\#}(u, \widetilde{v}) \geqslant \inf _{u \in X_{k}} J_{k}^{\#}(u, v)
\end{aligned}
$$

and (2.6) follows.

Now we show that for any $v \in X_{k}^{d}$

$$
\inf _{u \in X_{k}} J_{k}^{\#}(u, v)=J_{D_{k}}(v)
$$

Fix $v \in X_{k}^{d}$.

$$
\begin{aligned}
& \inf _{u \in X_{k}} J_{k}^{\#}(u, v)=-\sup _{u \in X_{k}}\left\{\int_{\Omega} \nabla u(x) v(x) d x-\int_{\Omega} V(x, \nabla u(x)) d x\right\}+\int_{\Omega}\left(F^{k}\right)^{*}(x,-\operatorname{div} v(x)) d x \\
& \geqslant-\sup _{f \in L^{D(x)}(\Omega)}\left\{-\int_{\Omega} f(x) v(x) d x-\int V(x, f(x)) d x\right\} \\
&+\int_{\Omega}\left(F^{k}\right)^{*}(x,-\operatorname{div} v(x)) d x \\
&=-\int_{\Omega} V^{*}(x, v(x)) d x+\int_{\Omega}\left(F^{k}\right)^{*}(x,-\operatorname{div} v(x)) d x=J_{D_{k}}(v) .
\end{aligned}
$$

On the other hand, there exist $u_{p}, \tilde{u}_{p} \in X_{k}$, related by (2.4), such that

$$
\begin{aligned}
-\operatorname{div} v(x) & =F_{u}^{k}(x, u(x)) \\
v(x) & =\mathcal{V}\left(x, \tilde{u}_{p}(x)\right)
\end{aligned}
$$


Therefore

$$
\begin{aligned}
J_{D_{k}}(v) & =\int_{\Omega}\left(F^{k}\right)^{*}(x,-\operatorname{div} v(x)) d x-\int_{\Omega} V^{*}(x, v(x)) d x \\
& =\int_{\Omega}\left(F^{k}\right)^{*}(x,-\operatorname{div} v(x)) d x-\int_{\Omega} v(x) \nabla \widetilde{u}_{p}(x)+\int_{\Omega} V(x, \nabla \tilde{u}(x)) d x \\
& =J_{k}^{\#}(\tilde{u}, v) \leqslant \inf _{u \in X_{k}} J_{k}^{\#}(u, v)
\end{aligned}
$$

Hence (2.7) holds. This and (2.6) provide

$$
\inf _{u \in X_{k}} J_{k}(x)=\inf _{v \in X_{k}^{d}} J_{D_{k}}(v)
$$

We shall use the duality results to derive necessary conditions for the existence of solutions to (1.1).

Theorem 2.5. (Variational Principle) Assume (F1)-(F5) and that for any $k=0,1, \ldots$ there exists $\bar{u}_{k} \in X_{k}$ such that $-\infty<J_{k}\left(\bar{u}_{k}\right)=\inf _{u \in X_{k}} J_{k}(u)<\infty$. Then there exist $\bar{v}_{k} \in X_{k}^{d}, k=0,1, \ldots$ such that

$$
\begin{gathered}
-\operatorname{div} \bar{v}_{k}(x)=F_{u}^{k}\left(x, \bar{u}_{k}(x)\right), \\
\mathcal{V}\left(x, \nabla u_{k}(x)\right)=\bar{v}_{k}(x) .
\end{gathered}
$$

\section{Moreover}

$$
\inf _{v \in X_{k}^{d}} J_{D_{k}}(v)=J_{D_{k}}\left(\bar{v}_{k}\right)=J_{k}\left(\bar{u}_{k}\right)=\inf _{u \in X_{k}} J_{k}(u) .
$$

Proof: Since $\bar{u}_{k} \in X_{k}$, we may take $\bar{v}_{k} \in X_{k}^{d}$ such that

$$
-\operatorname{div} \bar{v}_{k}(x)=F_{u}^{k}\left(x, \bar{u}_{k}(x)\right)
$$

Thus (2.8) holds. By (2.8) and by the Fenchel-Young inequality we have

$$
\begin{aligned}
J_{k}\left(\bar{u}_{k}\right)= & \int_{\Omega} V\left(x, \nabla \bar{u}_{k}(x)\right) d x-\int_{\Omega} F^{k}\left(x, \bar{u}_{k}(x)\right) d x \\
= & -\int_{\Omega}\left(-\operatorname{div} \bar{v}_{k}(x)\right) \nabla \bar{u}_{k}(x) d x+\int_{\Omega}\left(F^{k}\right)^{*}\left(x,-\operatorname{div} \bar{v}_{k}(x)\right) d x \\
& \quad+\int_{\Omega} V\left(x, \nabla \bar{u}_{k}(x)\right) d x \\
& \geqslant-\int_{\Omega} V^{*}\left(x, \bar{v}_{k}(x)\right) d x+\int_{\Omega}\left(F^{k}\right)^{*}\left(x,-\operatorname{div} \bar{v}_{k}(x)\right) d x=J_{D_{k}}\left(\bar{v}_{k}\right) .
\end{aligned}
$$

Hence $J_{k}\left(\bar{u}_{k}\right) \geqslant J_{D_{k}}\left(\bar{v}_{k}\right)$. By Theorem 2.4 it follows that

$$
J_{k}\left(\bar{u}_{k}\right)=\inf _{u \in X_{k}} J_{k}(u)=\inf _{v \in X_{k}^{d}} J_{D_{k}}(v) \leqslant J_{D_{k}}\left(\bar{v}_{k}\right)
$$


Hence $J_{k}\left(\bar{u}_{k}\right)=J_{D_{k}}\left(\bar{v}_{k}\right)$ and

$$
\begin{aligned}
\int_{\Omega} V\left(x, \nabla \bar{u}_{k}(x)\right) d x & -\int_{\Omega} F^{k}\left(x, \bar{u}_{k}(x)\right) d x \\
& =-\int_{\Omega} V^{*}\left(x, \bar{v}_{k}(x)\right) d x+\int_{\Omega}\left(F^{k}\right)^{*}\left(x,-\operatorname{div} \bar{v}_{k}(x)\right) d x .
\end{aligned}
$$

By the above and (2.8) it follows that

$$
V\left(x, \nabla \bar{u}_{k}(x)\right)+V^{*}\left(x, \bar{v}_{k}(x)\right)=\bar{v}_{k}(x) \nabla \bar{u}_{k}(x) .
$$

Hence (2.9) holds. Assertion (2.10) follows by Duality Principle and since $J_{k}\left(\bar{u}_{k}\right)$ $=J_{D_{k}}\left(\bar{v}_{k}\right)$.

\section{THE EXISTENCE OF SOLUTIONS}

Theorem 3.1. Assume (F1)-(F5). For all $k=0,1,2 \ldots$ there exists $\left(u_{k}, v_{k}\right)$ $\in X_{k} \times X_{k}^{d}$ such that

$$
\begin{gathered}
-\operatorname{div} v_{k}(x)=F_{u}^{k}\left(x, u_{k}(x)\right) \\
\nu\left(x, \nabla u_{k}(x)\right)=v_{k}(x)
\end{gathered}
$$

Moreover

$$
\inf _{v \in X_{k}^{d}} J_{D_{k}}(v)=J_{D_{k}}\left(v_{k}\right)=J_{k}\left(u_{k}\right)=\inf _{u \in X_{k}} J_{k}(u)
$$

Proof: First we show that $J_{k}$ is bounded from below on $X_{k}$. Let us fix $k=0,1, \ldots$ By convexity of $V, F_{u}^{k},(F 1)$, and integration by parts we get for any $u_{k} \in X_{k}$

$$
\int_{\Omega} V\left(x, \nabla u_{k}(x)\right) d x \geqslant \int_{\Omega} V(x, 0) d x+\int_{\Omega} \mathcal{V}(x, 0) \nabla u_{k}(x) d x
$$

By (F4) it follows that $\int_{\Omega} V\left(x, \nabla u_{k}(x)\right) d x$ is bounded from below on $X_{k}$.

Moreover, by Lemma 2.3 we obtain the boundedness of $\int_{\Omega} F^{k}\left(x, u_{k}(x)\right) d x$. As a consequence $J_{k}$ is bounded from below on $X_{k}$.

By the definition of $X_{k}$ it follows that it is weakly compact in $W^{1, p(x)}(\Omega)$. Therefore we can find a minimising sequence $\left\{u_{k}^{(n)}\right\}$ for $J_{k}$ in $X_{k}$ which is, possibly up to subsequence, convergent weakly in $W^{1, p(x)}(\Omega)$, strongly in $L^{p(x)}(\Omega)$ and thus strongly in $L^{p^{-}}(\Omega)$. Therefore it is convergent almost everywhere. We denote its limit by $u_{k}^{(0)}$. Therefore

$$
\lim _{n \rightarrow \infty} \int_{\Omega} F^{k}\left(x, u_{k}^{(n)}(x)\right) d x=\int_{\Omega} F^{k}\left(x, u_{k}^{(0)}(x)\right) d x .
$$


Moreover, since $\left\{\nabla u_{k}^{(n)}\right\}$ is convergent weakly in $L^{p(x)}(\Omega)$ and $V$ is convex and lower semicontinuous we get

$$
\liminf _{n \rightarrow \infty} \int_{\Omega} V\left(x, \nabla u_{k}^{(n)}(x)\right) d x \geqslant \int_{\Omega} V\left(x, \nabla u_{k}^{(0)}(x)\right) d x .
$$

Thus $J_{k}$ is lower semicontinuous on $X_{k}$. Consequently, for any $k=0,1,2, \ldots$ one can find $u_{k} \in X_{k}$ such that $J_{k}\left(u_{k}\right)=\inf _{u \in X_{k}} J_{k}(u)$. By Theorem 2.5 it follows that there exists $v_{k} \in X_{k}^{d}$ satisfying (3.1). As in Theorem 2.5 we assert that (3.2) and (3.3) also hold. $\quad$

COROLlary 3.2. For all $k=0,1,2, \ldots$ there exists $u_{k} \in X_{k}$ such that

$$
\begin{aligned}
-\operatorname{div}\left(\mathcal{V}\left(x, \nabla u_{k}(x)\right)\right) & =F_{u}^{k}\left(x, u_{k}(x)\right), \\
u_{k} \mid \partial \Omega & =0, \\
J_{k}\left(u_{k}\right) & =\inf _{u \in X_{k}} J_{k}(u) .
\end{aligned}
$$

Moreover $-\operatorname{div}\left(\nu\left(\cdot, \nabla u_{k}(\cdot)\right)\right) \in L^{\infty}(\Omega)$ and

$$
\left\|\nabla u_{k}\right\|_{L^{p(x)}(\Omega)} \leqslant \frac{d_{k}}{C_{1} C_{2}},\left|u_{k}(x)\right| \leqslant d_{k}
$$

\section{StaBility OF SOLUTIONS}

ThEOREM 4.1. Assume (F1)-(F5) and that for every $u \in W_{0}^{1, p(x)}(\Omega)$ there is a subsequence $\left\{k_{i}\right\}$ such that

$$
\lim _{i \rightarrow \infty} F_{u}^{k_{i}}(x, u(x))=F_{u}^{0}(x, u(x))
$$

weakly in $L^{\infty}(\Omega)$. Then for each $k=0,1,2, \ldots$ there exists a solution $u_{k}$ to problem (1.1). There exists a subsequence $\left\{u_{k_{n}}\right\}_{n=1}^{\infty}$ of the sequence $\left\{u_{k}\right\}_{k=1}^{\infty}$ and $\bar{u} \in W_{0}^{1, p(x)}(\Omega)$ such that

$$
u_{k_{n}} \rightarrow \bar{u} \in X_{0}, \text { strongly in } W_{0}^{1, p(x)}(\Omega)
$$

and

$$
\begin{gathered}
-\operatorname{div}(\mathcal{V}(x, \nabla \bar{u}(x)))=F_{u}^{0}(x, \bar{u}(x)) \\
\left.\bar{u}(x)\right|_{\partial \Omega}=0
\end{gathered}
$$

Moreover

$$
J_{0}(\bar{u})=\inf _{u \in X_{0}} J_{0}(\bar{u})
$$

Proof: By Corollary 3.2 it follows that for each $k=0,1,2, \ldots$ there exists $u_{k} \in W_{0}^{1, p(x)}(\Omega)$ satisfying (1.1). Due to the fact that $X_{k} \subset X_{0}$ it follows that the sequence $\left\{\nabla u_{k}\right\}_{k=1}^{\infty}$ is bounded in $L^{p(x)}(\Omega)$ and we may choose a weakly convergent subsequence 
in $W_{0}^{1, p(x)}(\Omega)$ which up to a subsequence may be assumed to be strongly convergent in $L^{p^{-}}(\Omega)$ and thus convergent almost everywhere. We denote its limit by $\bar{u}$. By the assumption we may take a subsequence $\left\{k_{i}\right\}_{i=1}^{\infty}$ such that $\lim _{i \rightarrow \infty} F_{u}^{k_{i}}(x, \bar{u}(x))=F_{u}^{0}(x, \bar{u}(x))$ weakly in $L^{\infty}(\Omega)$. We denote all the resulting subsequences by the subscript $k$ for simplicity. Due to (2.3) we obtain

$$
\underset{x \in \Omega}{\operatorname{esssup}}\left|F_{u}^{k}(x, \bar{u}(x))-F_{u}^{0}(x, \bar{u}(x))\right| \leqslant \frac{2 a_{0} d_{0}}{C_{1} C_{2} C_{S}}
$$

This and the definition of $X_{k}$ imply that $\left\{-\operatorname{div}\left(\nu\left(\cdot, \nabla u_{k}(\cdot)\right)\right)\right\}_{k=1}^{\infty}$ is, up to a subsequence, weakly convergent in $L^{q(x)}(\Omega)$ to a certain function $d \in L^{q(x)}(\Omega)$. Thus

$$
\int_{\Omega}\left\langle-\operatorname{div}\left(\mathcal{V}\left(x, \nabla u_{k}(x)\right)\right)-\operatorname{div}(\mathcal{V}(x, \nabla \bar{u}(x))), u_{k}(x)-\bar{u}(x)\right\rangle d x \rightarrow 0 .
$$

Hence and by the fact that the operator $-\operatorname{div}\left(\mathcal{V}\left(\cdot, \nabla u_{k}(\cdot)\right)\right)$ has $\left(S_{+}\right)$property it follows that $\left\{\nabla u_{k}\right\}_{k=1}^{\infty}$ is strongly convergent in $W_{0}^{1, p(x)}(\Omega)$.

We shall next prove that

$$
-\operatorname{div}(\mathcal{V}(x, \nabla \bar{u}(x)))=F_{u}^{0}(x, \bar{u}(x)) .
$$

By the convexity of $F^{k}$ we get for any $u \in W_{0}^{1, p(x)}(\Omega)$

$$
\int_{\Omega}\left\langle F_{u}^{k}\left(x, u_{k}(x)\right)-F_{u}^{k}(x, u(x)), u_{k}(x)-u(x)\right\rangle d x \geqslant 0 .
$$

Corollary 3.2 provides

$$
\int_{\Omega}\left\langle-\operatorname{div}\left(\mathcal{V}\left(x, \nabla u_{k}(x)\right)\right)-F_{u}^{k}(x, u(x)), u_{k}(x)-u(x)\right\rangle d x \geqslant 0 .
$$

Since $u_{k} \rightarrow \bar{u}$ strongly in $L^{p(x)}(\Omega)$ and $F_{u}^{k}(\cdot, u(\cdot)) \rightarrow F_{u}^{0}(\cdot, u(\cdot))$ weakly in $L^{q(x)}(\Omega)$ we easily get that

$$
\int_{\Omega}\left\langle-F_{u}^{k}(x, u(x)), u_{k}(x)-u(x)\right\rangle d x \rightarrow \int_{\Omega}\left\langle-F_{u}^{0}(x, u(x)), \bar{u}(x)-u(x)\right\rangle d x .
$$

Moreover

$$
\begin{aligned}
\int_{\Omega}\langle & \left.-\operatorname{div}\left(\mathcal{V}\left(x, \nabla u_{k}(x)\right)\right),-u(x)\right\rangle d x=\int_{\Omega}\left\langle\mathcal{V}\left(x, \nabla u_{k}(x)\right),-\nabla u(x)\right\rangle d x \\
\rightarrow \int_{\Omega}\langle\mathcal{V}(x, \nabla \bar{u}(x)),-\nabla u(x)) d x & =\int_{\Omega}\langle-\operatorname{div}(\mathcal{V}(x, \nabla \bar{u}(x))),-u(x)\rangle d x .
\end{aligned}
$$

We further observe that

$$
\begin{aligned}
\int_{\Omega}\left\langle-\operatorname{div}\left(\mathcal{V}\left(x, \nabla u_{k}(x)\right)\right), u_{k}(x)\right\rangle d x & =\int_{\Omega} \mathcal{V}\left(x, \nabla u_{k}(x)\right) \nabla u_{k}(x) d x \\
& \rightarrow \int_{\Omega}-\operatorname{div}(\mathcal{V}(x, \nabla \bar{u}(x))) \nabla \bar{u}(x) d x
\end{aligned}
$$


Hence

$$
\int_{\Omega}\left\langle-\operatorname{div}(\nu(x, \nabla \bar{u}(x)))-F_{u}^{0}(x, u), \bar{u}(x)-u(x)\right\rangle d x \geqslant 0
$$

for any $u \in W_{0}^{1, p(x)}(\Omega)$.

Now we apply the Minty "trick" that is, we consider the points $\bar{u}+t u$, where $u \in W_{0}^{1, p(x)}(\Omega),|u(x)| \leqslant d_{0}$ on $\Omega$ and $t>0$ are such that $\bar{u}(x)+t u(x) \in I$ almost everywhere. By inequality (4.2) we obtain

$$
\int_{\Omega}\left\langle-\operatorname{div}(\mathcal{V}(x, \nabla \bar{u}(x)))-F_{u}^{0}(x, \bar{u}(x)+t u(x)), u(x)\right\rangle d x \leqslant 0
$$

Since the function $t \mapsto F^{0}(\cdot, \bar{u}(\cdot)+t u(\cdot))$ is convex it follows that its derivative $t \mapsto \int_{\Omega}\left\langle F_{u}^{0}(x, \bar{u}(x)+t u(x)), u(x)\right\rangle d x$ is continuous at any sufficiently small $t$. Hence for any $u \in W_{0}^{1, p(x)}(\Omega)$ satisfying the above conditions

$$
\begin{aligned}
0 \geqslant \lim _{t \rightarrow 0} \int_{\Omega}\langle-\operatorname{div}(\nu(x, \nabla \bar{u}(x))) & \left.-F_{u}^{0}(x, \bar{u}(x)+t u(x)), u(x)\right) d x \\
= & \int_{\Omega}\left(-\operatorname{div}(\mathcal{V}(x, \nabla \bar{u}(x)))-F_{u}^{0}(x, \bar{u}(x)), u(x)\right) d x .
\end{aligned}
$$

Since $-\operatorname{div}(\mathcal{V}(\cdot, \nabla \bar{u}(\cdot)))-F_{u}^{0}(\cdot, \bar{u}(\cdot)) \in L^{p(x)}(\Omega)$ we obtain $(4.1)$.

\section{EXAMPLE}

Consider the problem

$$
\begin{gathered}
-\operatorname{div}\left(\varphi\left(x,|\nabla u|^{p(x)-1}\right)|\nabla u|^{p(x)-2} \nabla u\right)=F_{u}^{k}(x, u) \\
\left.u\right|_{\partial \Omega}=0, u \in W_{0}^{1, p(x)}(\Omega)
\end{gathered}
$$

where $\Omega \subset \mathbb{R}^{N}$ is a bounded region satisfying (F1), $F$ is subject to F1, F2, F3 and $\varphi$ satisfies

O1 $\varphi: \Omega \times \mathbb{R} \rightarrow \mathbb{R}$ is a Caratheodory function; there exist constants $a_{0}, a_{1}>0$ such that for almost everywhere $x \in \Omega$ and $a_{0} \leqslant \varphi_{k}(x, a) \leqslant a_{1}$ for all $a \in \mathbb{R}_{+} ;$there exists a constant $m>0$ such that

$$
\varphi(x, a) a-\varphi(x, b) b \geqslant m(a-b)
$$

for all $a \geqslant b, a, b \in \mathbb{R}$ and almost everywhere $x \in \Omega$.

We observe that assumptions F4, F5, F6 are obviously satisfied. Indeed, exactly as in [9] we may prove that operator

$$
-\operatorname{div}\left(\varphi\left(\cdot,|\nabla u(\cdot)|^{p(\cdot)-1}\right)|\nabla u(\cdot)|^{p(\cdot)-2} \nabla u(\cdot)\right)
$$


is monotone and by its definition it follows that it is radially continuous and coercive. We need to write $V$ explicitly

$$
\int_{\Omega} V(x, h(x)) d x=\int_{\Omega} \int_{0}^{|h(x)|} \varphi_{k}\left(x, s^{p(x)-1}\right) s^{p(x)-1} d s d x .
$$

5.1. EXISTENCE We take up an following example

$$
\begin{gathered}
-\operatorname{div}\left(\varphi\left(x,|\nabla u|^{p(x)-1}\right)|\nabla u|^{p(x)-2} \nabla u\right)=|x|^{2} \cdot|u(x)|^{\alpha(x)-1} u(x)+|x|^{2}, \\
\left.u\right|_{\partial \Omega}=0, u \in W_{0}^{1, p(x)}(\Omega)
\end{gathered}
$$

where $\Omega=B(0,1 / 2)$ is a ball in $\mathbb{R}^{3}, p \in C(\bar{\Omega}), p^{-}>3$. We also assume $\alpha(x) \geqslant \alpha^{-}>p^{+}$ and that $\alpha$ has an upper bound $\alpha^{+}>2$.

We shall show that (F1)-(F3) hold. Here

$$
F(x, u)=\frac{1}{\alpha(x)+1}|x|^{2} \cdot|u(x)|^{\alpha(x)+1}+|x|^{2} \cdot u(x)
$$

and it is Caratheodory function, convex in $u$. Of course $F_{u}$ is also Caratheodory, such that $F_{u}(x, 0) \neq 0$ almost everywhere on $\Omega$ and $F_{u}(\cdot, w) \in L^{\infty}(\Omega)$ for any fixed $w \in \mathbb{R}$. Moreover, the functions $x \mapsto F(x, 0)$ and $x \mapsto F^{*}(x, 0)$ are integrable on $\Omega$. Thus (F2) and (F3) hold. Clearly $\operatorname{vol}(\Omega) \leqslant\left(1 / p^{-}+1 / q^{-}\right)^{-1}$.

Assume that $C_{1} C_{2}>1$ and

$$
\text { O2 } a_{0}>\left(C_{1} C_{2} C_{S}\right) / 2 \text { for all } x \in \Omega \text {. }
$$

To conclude that (F1) is satisfied we only need to show that there exists a constant $d \geqslant C_{1} C_{2}$ such that inequalities in (2.3) hold. We have

$$
\begin{gathered}
C_{1} C_{2} C_{S} \text { ess sup }\left|F_{u}(x, d)\right|=C_{1} C_{2} C_{S} \frac{d^{\alpha^{+}}+1}{4}, \\
C_{1} C_{2} C_{S} \underset{x \in \Omega}{\operatorname{ess} \sup _{x}}\left|F_{u}(x,-d)\right|=C_{1} C_{2} C_{S} \frac{d^{\alpha^{+}}-1}{4}
\end{gathered}
$$

Therefore we need to find $d$ satisfying $d^{\alpha^{+}}+1 \leqslant\left(4 a_{0} d\right) /\left(C_{1} C_{2} C_{S}\right)$. Since $d \geqslant 1$ we have that $d^{\alpha^{+}}+1 \leqslant 2 d^{\alpha^{+}}$and since

$$
2 d^{a^{+}}=\frac{4 a_{0} d}{C_{1} C_{2} C_{S}}
$$

when $d=\left(\left(2 a_{0}\right) /\left(C_{1} C_{2} C_{S}\right)\right)^{1 /\left(a^{+}-1\right)}$ we assert by $(\mathrm{O} 2)$ that $d \geqslant 1$ and inequalities in (2.3) hold. Finally we put

$$
d=\max \left\{C_{1} C_{2},\left(\frac{2 a_{0}}{C_{1} C_{2} C_{S}}\right)^{1 /\left(a^{+}-1\right)}\right\} .
$$

We may now construct the set $X$ which reads

$$
X=\left\{u \in W_{0}^{1, p(x)}(\Omega):\|\nabla u\|_{p(x)} \leqslant \frac{d}{C_{1} C_{2}},|u(x)| \leqslant d \text { for all } x \in \Omega\right\},
$$

where $d$ is given above. Corollary 3.2 provides existence of solution to (5.2). 
5.2. Stability We consider the problem

$$
\begin{gathered}
-\operatorname{div}\left(\varphi\left(x,|\nabla u|^{p(x)-1}\right)|\nabla u|^{p(x)-2} \nabla u\right)=|x|^{2} \cdot|u(x)|^{\alpha_{k}(x)-1} u(x)+|x|^{2}, \\
\left.u\right|_{\partial \Omega}=0, u \in W_{0}^{1, p(x)}(\Omega),
\end{gathered}
$$

with $\Omega=B(0,(1 / 2)) \subset \mathbb{R}^{3}, p$ as above, $\alpha_{k}(x) \geqslant \alpha_{k}^{-}>p^{+}$and with an upper bound $\alpha_{k}^{+}>2$ for each $k=0,1, \ldots$.

As previously the assumptions (F1)-(F6) are satisfied and for each $k=0,1, \ldots$ there exists a solution to (5.3). We assume (O2) and

$$
\text { O3 } \alpha_{1}^{+}>\alpha_{2}^{+}>\cdots>\alpha_{k}^{+}>\alpha_{k+1}^{+}>\cdots>\alpha_{0}^{+}>2 \text {. }
$$

We obtain as previously that $d_{k}=\max \left\{C_{1} C_{2},\left(\left(2 a_{0}\right) /\left(C_{1} C_{2} C_{S}\right)\right)^{1 /\left(\alpha_{k}^{+}-1\right)}\right\}$ for every $k=0,1, \ldots$ and by $(\mathrm{O} 3)$ it is an nondecreasing sequence. Consequently the sets

$$
X_{k}=\left\{u \in W_{0}^{1, p(x)}(\Omega):\|\nabla u\|_{p(x)} \leqslant \frac{d_{k}}{C_{1} C_{2}},|u(x)| \leqslant d_{k} \text { for all } x \in \Omega\right\}
$$

form into an nondecreasing sequence $X_{1} \subseteq X_{2} \subseteq \ldots \subseteq X_{0}$. By Theorem 4.1 it follows that the solutions $u_{k}$ of (5.3) up to a subsequence converge to a certain $u_{0} \in X_{0}$ being a solution to

$$
\begin{gathered}
-\operatorname{div}\left(\varphi\left(x,|\nabla u|^{p(x)-1}\right)|\nabla u|^{p(x)-2} \nabla u\right)=|x|^{2} \cdot|u(x)|^{\alpha_{0}(x)-1} u(x)+|x|^{2}, \\
\left.u\right|_{\partial \Omega}=0, u \in W_{0}^{1, p(x)}(\Omega) .
\end{gathered}
$$

\section{REFERENCES}

[1] R.A. Adams, Sobolev spaces (Academic Press, New York, 1975).

(2) C.O. Alves and A.S. Marco, 'Existence of solutions for a class of problems in $\mathbb{R}^{N}$ involving the $\mathrm{p}(\mathrm{x})$-Laplacian', in Contributions to nonlinear analysis, Nonlinear Differential Equations Appl. 66 (Birkhuser, Basel, 2006), pp. 17-32.

[3] J. Chabrowski and Y. Fu, 'Existence of solutions for $p(x)$-Lapacian problem on a bounded domain', J. Math. Anal. Appl. 306 (2005), 604-618.

[4] I. Ekeland and R. Temam, Convex analysis and variational problems (North-Holland, Amsterdam, 1976).

[5] X.L. Fan and X.Y. Han, 'Existence and multiplicity of solutions for $\mathrm{p}(\mathrm{x})$-Laplacian equations in $\mathbb{R}^{N}$, Nonlinear Anal. 59 (2004), 173-188.

[6] X.L. Fan and D. Zhao, 'Sobolev embedding theorems for Spaces $W^{k, p(x)}(\Omega)$ ', J. Math. Anal. Appl. 262 (2001), 749-760.

[7] X.L. Fan and D. Zhao, 'Existence of solutions for $p(x)$-Lapacian Dirichlet problem', Nonlinear Anal. 52 (2003), 1843-1852.

[8] X.L. Fan and D. Zhao, 'On the Spaces $L^{p(x)}(\Omega)$ and $W^{k, p(x)}(\Omega)$ ', J. Math. Anal. Appl. 263 (2001), 424-446.

[9] H. Gajewski, K. Groeger and K. Zacharias, Nichtlineare Operatorgleichungen und operatordifferentialgleichungen (Akademie-Verlag, Berlin, 1974). 
[10] M. Galewski, 'Stability of solutions for an abstract Dirichlet problem', Ann. Polon. Math. 83 (2004), 273-280.

[11] M. Galewski, 'New variational method for $p(x)$-Laplacian equation', Bull. Austral. Math. Soc. 72 (2005), 53-65.

[12] D. Idczak, 'Stability in semilinear problems', J. Differential Equations 162 (2000), 64-90.

[13] M. Ruzicka, 'Electrorheological fluids: modelling and mathematical theory', in Lecture Notes in Mathematics 1748 (Springer-Verlag, Berlin, 2000).

[14] S. Walczak, 'On the continuous dependance on parameters of solutions of the Dirichlet problem. Part I. Coercive Case, Part II. The Case of Saddle Points', Acad. Roy. Belg. Bull. Cl. Sci. (6) 6 (1995), 247-273.

[15] S. Walczak, 'Continuous dependance on parameters and boundary data for nonlinear P.D.E. coercive case', Differential Integral Equations 11 (1998), 35-46.

[16] Q.H. Zhang, 'Singularity of positive radial solutions for a class of $p(x)$-Laplacian equations', J. Lanzhou Univ. Nat. Sci. 36 (2000), 5-11.

[17] Q.H. Zhang, 'Existence of radial solutions for $\mathrm{p}(\mathbf{x})$-Laplacian equations in $\mathbb{R}^{N}, J$. Math. Anal. Appl. 315 (2006), 506-516.

[18] V.V. Zhikov, 'A veraging of functionals of the calculus of variations and elasticity theory', Math. USSR Izv. 29 (1987), 33-66.

Faculty of Mathematics

University of Lódź

Banacha 22

90-238 Lódź

Poland

e-mail: galewski@math.uni.lodz.pl

plo@math.uni.lodz.pl 